\title{
Étude Raman de l'effet de taille nanométrique des cristallites sur le diagramme de phase P-T de la zircone
}

\author{
P. Bouvier et G. Lucazeau
}

LEPMI, CNRS INPG, 1130 rue de la Piscine, BP. 75, 38402 Saint-Martin-d'Hères cedex, France

\begin{abstract}
The phase transformations of $7,13,18 \mathrm{~nm}$ nanocrystalline zirconia prepared by pyrosol technique and $10-15 \mathrm{~nm}$ zirconia prepared by oxidation of zirconium alloys have been monitored by Raman spectrometry. It is shown that for these zirconia, the tetragonal form can be obtained either under its metastable state at ambient conditions or from the monoclinic form by application of pressure below 4 GPa and with temperature increment smaller than $300 \mathrm{~K}$. The nanometric monoclinic-tetragonal equilibrium line is strongly down-shifted in temperature compared to literature data measured on usual polycrystalline zirconia. In addition, the monoclinic-orthorhombic equilibrium line is slightly shifted to higher pressure (i.e. $7 \mathrm{GPa}$ ). These results confirm that stresses can play a primordial role in the formation of tetragonal form in the oxidation process of zirconium alloys.
\end{abstract}

\section{INTRODUCTION}

L'effet de la taille nanométrique des cristallites sur la stabilisation des phases haute températures de la zircone a été étudié récemment $[1,2]$. Par exemple, pour des cristallites inférieures à $25 \mathrm{~nm}$, il est possible d'obtenir une zircone quadratique $(\mathrm{P} 4 / 2 \mathrm{nmc})$ à $300 \mathrm{~K}$.

Le diagramme P-T de la zircone massive est donné en partie dans la figure $1:$ les traits continus représentent les lignes d'équilibre entre les formes monocliniques (M), quadratique $(\mathrm{T})$ et orthorhombique $(\mathrm{O})$ pour une zircone micrométrique $[3,4]$ et les traits pointillés sont relatifs aux zircones nanométriques étudiées ici [5]. L'objectif de cette étude était d'examiner les conditions d'obtention de la forme quadratique sous les effets couplés de pression et de température dans des domaines compatibles avec ceux relatifs au développement des couches d'oxydation.

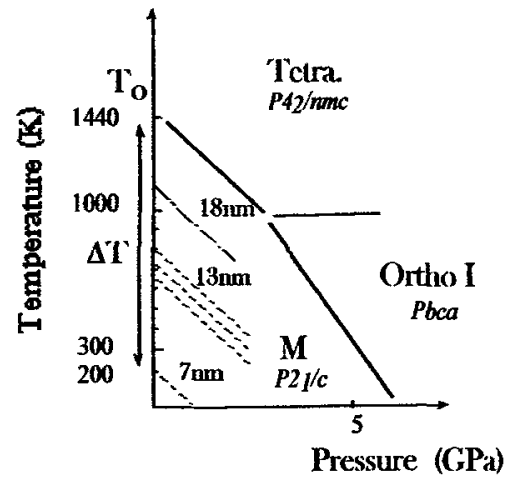

Fig 1: Diagramme de phases $P-T$ de $\mathrm{ZrO}_{2}$ indiquant les frontières en fonction de la taille

\section{CONDITIONS EXPERIMENTALES}

Échantillons: Les zircones 7, 13, $18 \mathrm{~nm}$ sont obtenues directement dans leur forme quadratique par un procédé pyrosol décrit dans [2]. Les zircones 10-15nm sont préparées par oxydation d'un alliage de zirconium, après dissolution du substrat métallique. Elles sont obtenues dans leur forme monoclinique.

Raman : Les spectres Raman sont obtenus sur un spectromètre XY Dilor avec la raie excitatrice $514.5 \mathrm{~nm}$ d'un laser argon. La cellule était placée sous un objectif $\times 201 f$.

Hautes pressions : Une cellule à enclume de diamant (DAC) BETSA a été utilisée pour les mesures sous pression à température ordinaire ou par chauffage laser. Le milieu transmetteur de 
pression est un mélange alcool-eau. Les mesures couplées pression-température sont réalisées sur une cellule DAC conçue et mise a disposition par le CEA Valduc. Le milieu transmetteur de pression est un gaz d'argon comprimé.

\section{RESULTATS}

\section{Température}

La figure 2 présente les spectres Raman traduisant la transformation réversible quadratiquemonoclinique vers $180 \mathrm{~K}$ pour une zircone $7 \mathrm{~nm}$. De même, fig. 3 , une zircone initialement dans sa forme quadratique ayant subi un chauffage à $600 \mathrm{~K}$ puis une trempe, retourne dans la forme monoclinique thermodynamiquement stable. Le passage vers la structure quadratique est observé pour cet échantillon pour une température de l'ordre de $600 \mathrm{~K}$.

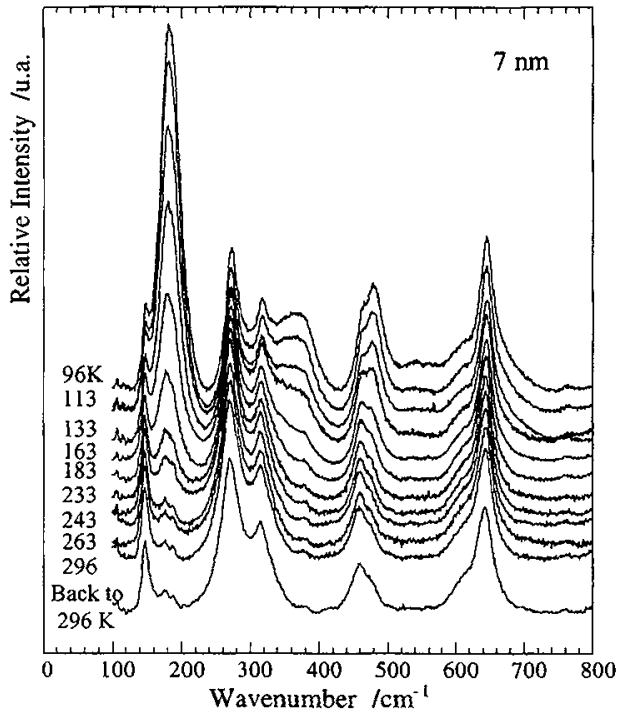

Fig. 2 :Spectres Raman mesurés sur le composé $7 \mathrm{~nm}$ traduisant la transition réversible $T-M$ à basse température. $L a$ transition n'est pas complète à $98 \mathrm{~K}$.

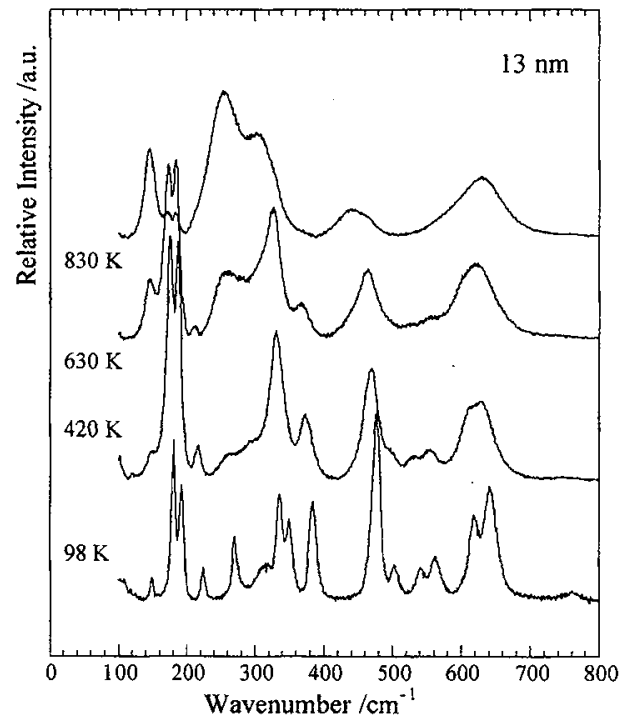

Fig. 3 : Spectres Raman mesurés sur le composé $13 \mathrm{~nm}$ traduisant la transition reversible $M-T$ à haute température. La forme monoclinique initiale est obtenue par une trempe à $98 \mathrm{~K}$.

Ces résultats indiquent que, à pression atmosphérique, les lignes d'équilibres des zircones 7 et 13 $\mathrm{nm}$ intersectent approximativement l'axe des températures vers 180 et $600 \mathrm{~K}$ respectivement. Les lignes d'équilibre correspondantes (en pointillé sur la fig. 1) sont tracées parallèlement à la ligne continue associée à une zircone massive. Cette représentation suppose que les grandeurs thermodynamiques associées à la transition monoclinique-quadratique des formes nanométriques sont conservées (relation de Clapeyron $\mathrm{dP} / \mathrm{dT}=\Delta \mathrm{S} / \Delta \mathrm{V}$ ). Par analogie la zircone $18 \mathrm{~nm}$ possède, sans doute, une température de transition supérieure à $1100 \mathrm{~K}$ mais il n'a pas été possible d'observer une transformation réversible à cette température dans la mesure où le grossissement des cristallites est rapide à cette température et provoque une déstabilisation de la forme quadratique. Il apparaît que la zircone $7 \mathrm{~nm}$ est dans son état stable à la température ambiante, c'est à dire $1200^{\circ}$ plus bas que pour le composé massif.

\section{Pression et pression-température}

L'ensemble des points explorés dans le diagramme P-T (fig. 4) sont indiqués par des cercles pour les mesures sous pression isostatique et par des triangles pour les mesures sous pression non- 
isostatique (poudre comprimée sans milieu transmetteur). Les spectres Raman reportés dans les figs. 5 et 6 illustrent les effets couplés de la pression et de la température sur une zircone $15 \mathrm{~nm}$ initialement monoclinique. Les spectres correspondent aux points numérotés dans le diagramme P-T de la figure 4. Les spectres (2) et (3) sont caractéristiques d'une zircone monoclinique. La forme orthorhombique est observée vers 7 $\mathrm{GPa}$, soit $3 \mathrm{GPa}$ au dessus de la pression critique habituellement mesurée pour une zircone microcristalline $[3,6]$. Les raies caractéristiques de la forme quadratique (repérées par des flèches dans la fig. 5) apparaissent progressivement à partir de $11 \mathrm{GPa}$ et $475 \mathrm{~K}$. L'abaissement de température seul ne permet pas de revenir à la forme orthorhombique pure (spectre (4) fig. 5). Les spectres (4-5)

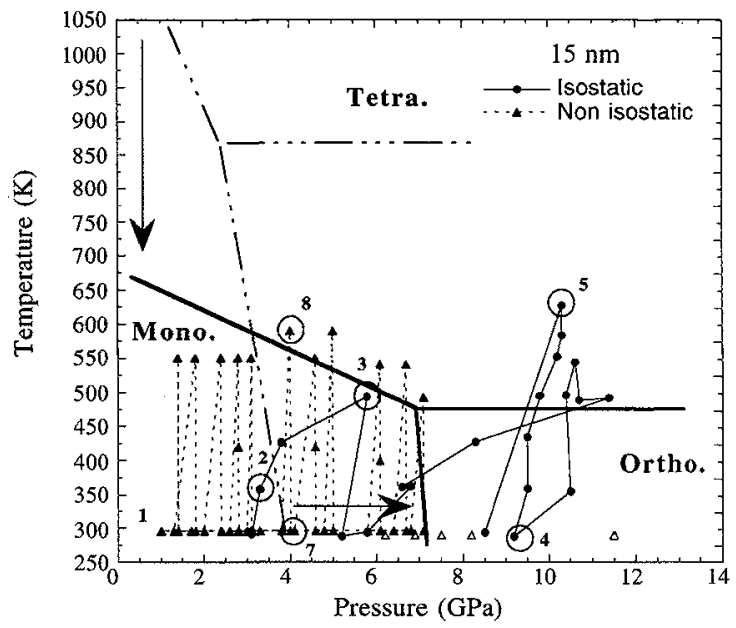
témoignent de la présence d'un

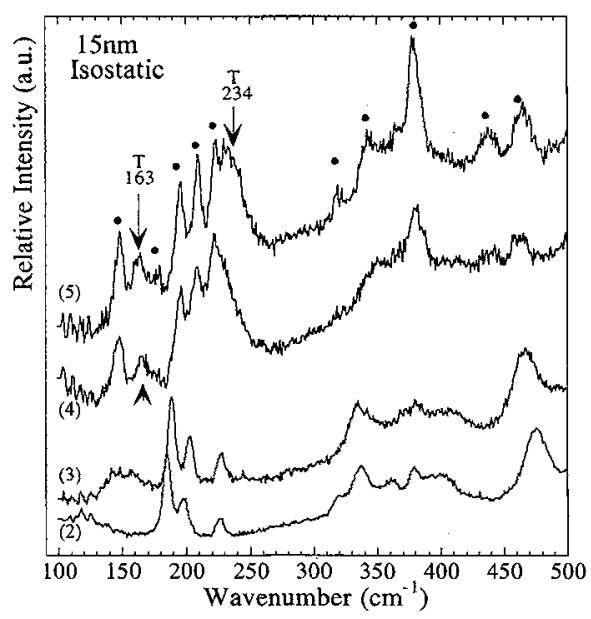

Fig 5 : Spectres Raman mesurés sous pression isostatique et en température. Les raies de la phase ortho. sont notées par des cercles noirs. Celles de la phase quadra. (T) par des flèches.
Fig. 4: Points explorés dans le diagramme P-T

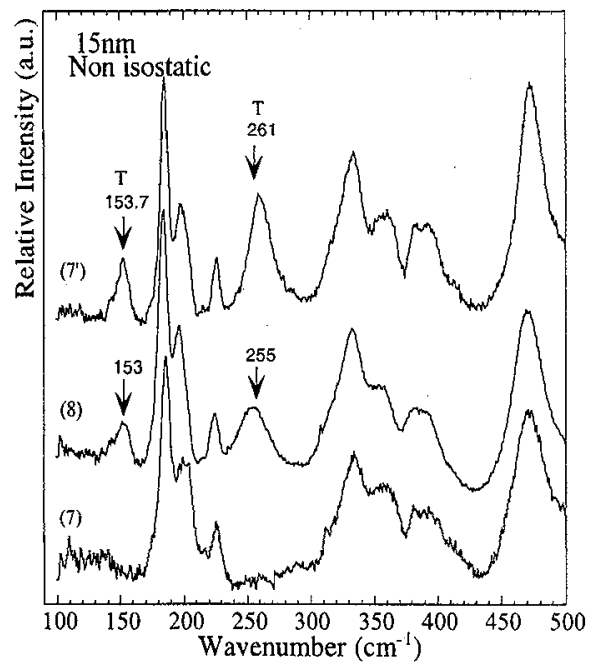

Fig. 6 : Spectres Raman mesurés sous pression non-isostatique et en température. Les raies de la phase quadra. sont repérées par des flèches. Les nombres d'onde sont indiqués.

mélange de phases orthorhombique et quadratique. Les mesures en pression non-isostatique permettent de préciser la position de la ligne monoclinique-quadratique. Ici encore l'abaissement de température seul ne permet pas de revenir à la forme monoclinique pure (spectre (7') fig. 6). Ce retour ne se fait qu'après relâchement complet de la pression. A noter que les lignes d'équilibre placées sur le diagramme de la fig. 4 sont très imprécises dans la mesure où les 
phases coexistent sur des domaines de $\mathrm{P}$ et $\mathrm{T}$ assez larges. Il convient d'admettre des incertitudes de l'ordre de $1 \mathrm{GPa}$ et $100 \mathrm{~K}$.

\section{DISCUSSION}

Les spectres Raman mesurés à basse température sur l'échantillon $7 \mathrm{~nm}$ permettent d'identifier deux composantes : (i) la première correspond aux raies de la forme quadratique ce qui montre que la transformation n'est pas complète, (ii) la seconde correspond aux raies larges centrées vers $180,340,470$ et $640 \mathrm{~cm}^{-1}$ qui sont attribuées à une forme monoclinique fortement désordonnée. Ce désordre est probablement dû à une conservation du caractère nanométrique des cristallites lors du processus de nucléation. Cette même transformation est observée sur le composé $13 \mathrm{~nm}$ à haute température $(600 \mathrm{~K})$. Ainsi à température ambiante la forme stable de la zircone $13 \mathrm{~nm}$ est monoclinique. L'état initial (quadratique) du composé est donc un état métastable. D'autre part, la transformation est complète pour le $13 \mathrm{~nm}$ tandis qu'elle n'est que partielle pour le $7 \mathrm{~nm}$. Cette différence est probablement due à une différence dans la dispersion des tailles de cristallites pour ces zircones. Des mesures sont actuellement en cours. L'abaissement de température de transition avec la taille des cristallites est sans doute lié à un effet d'énergie interfaciale des cristallites constituant les particules produites par pyrosol ou les grains constituant les couches d'oxydation. Dans l'hypothèse où la zone inter-cristallites est très désordonnée (amorphe), l'expression de Gibbs-Thomson peut être transposée à un équilibre solide-solide [7].

$$
\frac{\Delta T}{T^{0}}=\frac{2 V_{\alpha}}{\Delta H_{\alpha \beta}} \cdot \sigma \cdot \frac{1}{r}
$$

avec $\Delta T / T^{0}=\left(T-T^{0}\right) / T^{0}$ avec $T^{0}$ la température de transformation du cristal volumique, $V_{\alpha}$ le

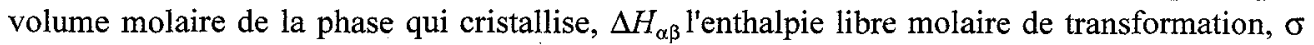
l'énergie interfaciale et $r$ le rayon du cristal (dans l'approximation sphérique). La variation de la température de transformation identifiée pour une taille égale à $7 \mathrm{~nm}$ permet de déterminer une énergie interfaciale égale à $496 \mathrm{~mJ} \cdot \mathrm{m}^{-2}$ avec $\Delta T / T^{0}=1$ et $T^{0}=1400 \mathrm{~K}, \Delta H_{\alpha \beta}=5.93 \mathrm{~kJ} . \mathrm{mole}^{-1}$ l'enthalpie de transformation monoclinique-quadratique et $V_{\alpha}=19.9 \mathrm{~cm}^{-3}$ le volume molaire de la phase quadratique ou monoclinique. Cette expression permet de retrouver une température de transformation de l'ordre de $600 \mathrm{~K}$ pour une taille égale à $13 \mathrm{~nm}$.

Les mesures couplées en pression et température permettent de montrer que la transformation monoclinique-orthorhombique est fortement déplacée vers les hautes pression pour les composés nanométriques. De plus, la pente $(\mathrm{dP} / \mathrm{dT})$ de la frontière monoclinique-quadratique est modifiée ce qui montre que les grandeurs thermodynamiques associées à cette transition sont dépendantes de la taille des cristallites. La métastabilité de la forme quadratique vis à vis de la température est confirmée par les mesures à haute pression. Par contre, cette zircone se retransforme totalement vers une forme monoclinique stable lorsque la pression est relâchée.

\section{CONCLUSION}

L'effet de taille des cristallites sur la stabilisation de la forme quadratique a été vérifié et discuté via la relation de Gibbs-Thomson. Les mesures couplées en pression et température ont permis de préciser les frontières entre phases. Ces observations confirment le rôle des contraintes de croissance à l'interface métal/oxyde dans la stabilisation d'une couche de quadratique proche de l'interface dans le processus d'oxydation des alliages de zirconium. 


\section{Remerciements}

Nous sommes reconnaissants à E. Djurado pour la préparation des zircones et à $B$. Sitaud et son équipe pour la mise à disposition de la cellule haute pression à température variable. $P$. Desré est également remercié pour son apport dans l'utilisation de l'équation de Gibbs-Thomson. J. Godlewski est remercié pour avoir suscité ce travail et pour les discussions dont il nous a fait bénéficier

\section{REFERENCES}

[1] R.C. Garvie, J. Phys. Chem. 69 (1965) 1238.

[2] E. Djurado, P. Bouvier, G. Lucazeau, J. Solid State Chem. 149, (2000) 399.

[3] G. Bocquillon and C. Susse, Rev. Int. Hautes Tempér. et Réfract. 6(1969) 263.

[4] S. Block, J.A.H. Da Jornada and G.J. Piermarini, J. Am. Ceram. Soc. 68 (1985) 497.

[5] P. Bouvier, thèse de doctorat Institut National Polytechnique de Grenoble (2000).

[6] H. Arashi and M. Ishigame, Phys. Stat. Sol. 71 (1982) 313.

[7] P.J. Desré, Phil. Mag. A 74 (1996) 103 et NanoStructured Materials 8 (1997) 687. 